\title{
TOWARDS LOCALIZATION IN LONG-RANGE CONTINUOUS INTERACTIVE ANDERSON MODELS
}

\author{
Victor CHULAEVSKY
}

Abstract. This paper is a follow-up of [8]. The main novelty is the proof of spectral and dynamical localization for a class of interactive Anderson models in Euclidean spaces with realistic, infinite-range inter-particle and media-particle potentials featuring a power-law decay at infinity. Specifically, we prove that in an energy interval near the bottom of the spectrum, the spectral measure is pure point with probability one, and the decay rate of the averaged eigenfunction correlators in this energy interval admits a summable power-law bound, the exponent of which grows along with the growth of the decay exponents of the potentials. The localized eigenfunctions admit a fractional-exponential bound on their decay rate. Earlier rigorous works on interactive Anderson models assumed the media-particle potential to be compactly supported.

Mathematics subject classification (2010): 60H25, 37K55.

Keywords and phrases: Multi-particle Anderson localization, infinite-range interactions, eigenvalue concentration estimates.

\section{REFERENCES}

[1] P. W. Anderson, Absence of diffusion in certain random lattices, Phys. Rev. 109 (5), 1492-1505 (1958).

[2] M. Aizenman, S. Warzel, Localization bounds for multiparticle systems, Commun. Math. Phys. 290, 903-934 (2009).

[3] J. M. Barbaroux, J. M. Combes And P. D. Hislop, Localization near band edges for random Schrödinger operators, Helv. Phys. Acta 70, 16-43 (1997).

[4] V. BEAUD, S. WARZEL, Low-energy Fock-space localization for attractive hard-core particles in disorder, Ann. Henri Poincaré 18 (10), 3143-3166 (2017).

[5] R. CARMONA, J. LACROIX, Spectral theory of random Schrödinger operators, Birkhäuser Boston Basel Berlin Inc. (1990).

[6] V. Chulaevsky, On resonances in disordered multi-particle systems, C.R.A.S. Acad. Sci. Paris, Ser. I 350, 81-85 (2012).

[7] V. Chulaevs Ky, From fixed-energy localization analysis to dynamical localization: An elementary path, J. Stat. Phys. 154, 1391-1429 (2014).

[8] V. ChUlAEVSKY, Efficient localization bounds in a continuous N-particle Anderson model with longrange interaction, Lett. Math. Phys. 106 (4), 509-533 (2016).

[9] V. Chulaevsky, Universality of smoothness of Density of States in arbitrary higher-dimensional disorder under non-local interactions I, From Viéte-Euler identity to Anderson localization, ArXiv:math-ph/1604.03312 (2016).

[10] V. Chulaevsky, A. Boutet de Monvel, Y. Suhov, Dynamical localization for a multi-particle model with an alloy-type external random potential, Nonlinearity 24 (5), 1451-1472 (2011).

[11] V. Chulaevsky, Y. Suhov, Multi-particle Anderson localisation: Induction on the number of particles, Math. Phys. Anal. Geom. 12, 117-139 (2009).

[12] V. Chulaevs Ky, Y. Suhov, Efficient Anderson localization bounds for large multi-partilce systems, J. Spec. Theory 7, 269-320 (2017).

[13] J. M. Combes, L. Thomas, Asymptotic behaviour of eigenfunctions for multi-particle Schrödinger operators, Commun. Math. Phys. 34, 251-263 (1973). 
[14] H. von Dreifus, A. KLeIn, A new proof of localization in the Anderson tight binding model, Commun. Math. Phys. 124, 285-299 (1989).

[15] H. VON DREIFUS, A. KLEIN, Localization for random Schrödinger operators with correlated potentials, Commun. Math. Phys. 140, 133-147 (1991).

[16] A. A. Elgart Klein, G. Stolz, Droplet localization in the random XXZ model and its manifestations, J. Phys. A 51 (1), 01LT02 (2018).

[17] A. Elgart, A. Klein, G. Stolz, Manifestations of dynamical localization in the disordered XXZ spin chain, Commun. Math. Phys. 361, 1083-1113 (2018).

[18] A. Elgart, A. Klein, G. Stolz, Many-body localization in the droplet spectrum of the random XXZ quantum spin chain, J. Funct. Anal. 275 (1), 211-258 (2018).

[19] A. Elgart, M. Tautenhahn, I. Veselić, Localization via fractional moments for models on $\mathbf{Z}$ with single-site potentials of finite support, J. Phys. A 43 (8), 474, 021 (2010).

[20] M. FAUSER, S. WARZEL, Multiparticle localization for disordered systems on continuous space via the fractional moment method, Rev. Math. Phys. 27 (4), 1550, 010 (2015).

[21] J. Fröhlich, F. Martinelli, E. Scoppola, T. SPEnCER, Constructive proof of localization in the Anderson tight binding model, Commun. Math. Phys. 101, 21-46 (1985).

[22] J. FRÖHLICH, T. SPENCER, Absence of diffusion in the Anderson tight binding model for large disorder or low energy, Commun. Math. Phys. 88, 151-184 (1983).

[23] F. Germinet, A. Klein, Bootstrap multiscale analysis and localization in random media, Commun. Math. Phys. 222, 415-448 (2001).

[24] F. Germinet, A. Klein, A characterization of the Anderson metal-insulator transport transition, Duke Math. J. 124 (2), 309-350 (2004).

[25] F. Germinet, A. Klein, New characterization of the region of complete localization for random Schrödinger operators, J. Stat. Phys. 122, 73-94 (2006).

[26] F. Germinet, A. KLEIn, A comprehensive proof of localization for continuous Anderson model with singular random potentials, J. Spec. Theory 15 (1), 55-143 (2013).

[27] W. Kirsch, P. Stollmann, G. Stolz, Anderson localization for random Schrödinger operators with long range interactions, Commun. Math. Phys. 195, 495-507 (1998).

[28] A. Klein, S. T. NGUYen, Bootstrap multiscale analysis for the multi-particle Anderson model, J. Stat. Phys. 151 (5), 938-973 (2013).

[29] A. Klein, S. T. NGUYen, Bootstrap multiscale analysis for the multi-particle continuous Anderson Hamiltonians, J. Spec. Theory 5 (2), 399-444 (2016).

[30] A. Klein, S. T. Nguyen, C. Rojas-Molina, Characterization of the metal-insulator transport transition for the two-particle Anderson model, Ann. Henri Poincaré 18, 2327-2365 (2017).

[31] F. MARTINELLI, E. SCOPPOLA, Remark on the absence of absolutely continuous spectrum for $d$ dimensional Schrödinger operators with random potential for large disorder or low energy, Commun. Math. Phys. 97, 465-471 (1985).

[32] P. Stollmann, Caught by disorder, Progress in Mathematical Physics, vol. 20, Birkhäuser Boston Inc., Boston, MA (2001), bound states in random media.

[33] M. Reed, B. Simon, Methods of modern mathematical physics, vol. 4, Academic Press, Inc., San Diego Boston Toronto (1979).

[34] F. Wegner, Bounds on the density of states in disordered systems, Z. Phys. B. Condensed Matter 44, 9-15 (1981). 